\title{
CATALOGO DE LAS ALGAS CONTINENTALES ESPAÑOLAS. \\ IV. CHLOROPHYCEAE WILLE IN WARMING 1884. PRASINOPHYCEAE T. CHRISTENSEN EX SILVA 1980
}

\author{
M. ALVAREZ COBELAS \& T. GALLARDO
}

RESUMEN: En este trabajo se refieren las algas continentales citadas para España hasta julio de 1981 pertenecientes a los grupos Chlorophyceae (557 taxones) y Prasinophyceae ( 9 taxones).

SUMAARY: A catalogue of Spanish inland algae belonging to Chlorophyceae (557 taxa) and Prasinophyceae ( 9 taxa) and found up to July 1981 is given.

\section{INTRODUCCION}

En este trabajo se recoge la parte cuarta del catálogo de algas continentales españolas, participando de todas las salvedades previas: sólo material publicado hasta 1981, simple inventario y no lista crítica, sinonimias de acuerdo con la bibliografía especializada y dispuestas al final del texto por orden alfabético. La literatura sobre la cual se basa esta cuarta parte, así como las tres porciones anteriores ya han sido publicadas previamente (Alvarez Cobelas, 1981, 1984a, 1984b; Alvarez Cobelas \& Estévez, 1982).

El grueso del presente estudio son las clorofíceas -palabra aquí empleada sin significado taxonómico alguno-, quizá el grupo de alga\$ sujeto a mayor investigación estructural en la actualidad. Como consecuencia de ella, los conceptos clásicos (desde Round, 1963, 1971, hacia atrás) de categorías superiores al género se encuentran sometidos a cambios radicales, la mayor parte de los cuales aún no pueden con- 
siderarse definitivos, aunque en líneas generales sigan las directrices de Stewart \& Mattox y su escuela. Recientemente (marzo de 1983), se ha celebrado un congreso internacional con el fin de consagrar dicho enfoque (Irvine \& John, 1985). Uno de los corolarios fundamentales de la nueva propuesta es el fraccionamiento de la antigua Clase Chlorophyceae Wille in Warming 1884 en tres : Chlorophyceae s. s. , Ulvophyceae y Charophyceae, éstas últimas todavía sin tipificar. Las alteraciones generadas por semejante propuesta resultan, a nuestro parecer, sumamente provisionales aún, motivo que nos induce a respetar el enfoque previo reflejado en las obras de Bourrelly $(1966,1972)$ y Silva (1980). La ordenación de nuestro trabajo seguirá, pues, la de estos últimos autores no porque la consideremos más acertada que la precedente, sino porque hasta ahora continúa siendo la más global y detallada de ambas. Otros motivos para su aceptación proviene de haber sido considerada como guía de los trabajos previos integrantes del presente catálogo, es decir: un motivo de indole unitaria.

Las publicaciones utilizadas para la sinonimización y puesta al día de la nomenclatura infrafamiliar han sido las siguientes:

Volvocales: Ettl (1970, 1975, 1979, 1983), Hüber-Pestalozzi (1961), Iyengar \& Desikachary (1981), Pocock (1960), Pringsheim (1969).

Tetrasporales: Fott (1972), Hoek (1963a), Lemmermann \& Brunnthaler (1915), Thompson (1956).

Chlorococcales: Archibald \& Bold (1970), Bigeard (1934), Fott \& Nováková (1969), Hegewald (1977), Hegewald \& Schnepf (1979), Hindak (1984), Jaag (1933), Komárek (1974), Komárek \& Fott (1983), KomárkováLegnerová (1969), Kováčik (1975), Lee \& Bold (1974), Lemmermann \& Brunnthaler (1915), Lewin (1984), Philippose (1967), Reháková (1969), Robinson \& Preston (1971), Schnepf et al. (1966), Smith \& Bold (1966), Sodomková (1972), Sulek (1969), Uherkovich (1966).

Ulotrichales: Hindak (1963), Lokhorst \& Vroman (1972, 1974a, b), Mattox \& Bold (1962), Pankow (1961), Printz (1964), Ramanathan (1964), Starmach (1972), Wichmann (1937).

Chaetophorales: Brand (1925), Cox \& Bold (1966), Herndon (1958), Nurul Islam (1963), Printz (1964), Starmach (1972), Wiel \& Reymond (1983).

Oedogoniales: Gemeinhardt (1939), Gonzalves (1981), Mrozińska-Webb (1969).

Trentepohliales: Printz (1964), Starmach (1972).

Sphaeropleales: Fritsch (1929).

Ulvales: Bliding (1963, 1968), Gallardo (1984), Vinogradova (1974).

Siphonocladales: Hamel (1931), Hoek (1963b), Starmach (1972).

Igualmente se han empleado como obras genéricas las de De Toni (1889) y Parke \& Dixon (1976). La lista no pretende en absoluto ser exhaustiva, entre otros motivos porque la bibliografía taxonómica de Cloroficeas es inmensa, cosa que no ocurre con la de Prasinofíceas, para las cuales hemos debido limitarnos a la consulta de Ettl (1983), Norris (1980) y Norris et al. (1980). 


\section{CATALOGO}

CHLOROPHYCEAE Wille in Warming

\section{VOLVOCALES}

\section{CHLAMYOOMONADACEAE}

Brachiomonas submarina Bohlin Carteria cordiformis (Carter) Dillw. Chlamydomonas acuta Korš. Chlamydomonas acutissima Pascher Chlamydomonas agloëformis Pascher Chlamydomas angulosa Dillw. Chlanydomonas applanata Pringsheim Chlamydomonas asymmetrica Korš. Chlamydomonas bacillus Pascher et Jahoda Chlamydomonas botryopara Rodhe et Skuja Chlamydomonas cavanillesiana González Guerrero (1)

Chlamydomonas conversa Korš.

Chlamydomas dangeardii Chmiliewski (2)

Chlamydomonas debaryana Gorosch.

Chlamydomonas gelatinosa Korš.

Chlamydomonas globosa Snow

Chlanydomonas gloeopara Rhode et Skuja

Chlamydomonas gloeophila Skuja

Chlanydomonas humicola Lucksch.

Chlamydomas incerta Pascher

Chlamydomonas pertusa Chodat

Chlamydomas peterfii Gerloff

Chlamydomonas planctonica ( 3 )

Chlanydomonas reinhardtii Dang.

Chlamydomonas simplex Pascher

Chlamydomonas snowiae Printz

var. pluristigma (Bristol) Gerloff

Chlamydomas sphagnophila Pascher

Chlamydomonas subcylindracea Korš.

Chlamydomonas umbonata Pascher

Chlorogonium minimum Playfair

Chloromonas acidophila (Nyg.) Gerloff

et Ettl
Haematococcus buetschlii Blochmann

Haematococcus pluvialis Flot. emend. Wille

Polytoma obtusum Pascher

Polytoma uvella Ehr.

Tetrablepharis multifilis (Klebs) Wille emend. Pascher

DUNANIELLACEAE

Asteromonas phacus Pascher

Collodictyon triciliatum Carter

Dunaliella salina Teodoresco

Dunaliella viridis Teodoresco PHACOTACEAE

Coccomonas orbicularis Stein

Phacotus lenticularis (Ehr.) Stein

Phacotus subglobosus Pascher

Sphaerellopsis fluviatilis (Stein) Pascher Sphaerellopsis gloeocystiformis (Dillw.) Gerloff

SPONDYLOMORACEAE

Spondylomorum quaeternarium Ehr. VOLVOCACEAE

Eudorina elegans Ehr.

Gonium formosum Pascher

Gonium pectorale 0.F. Müller

Gonium sociale (Duj.) Warming

Pandorina charkowiensis Korš.

Pandorina morum (O.F. Müller) Bory

Pleodorina californica Shaw

Stephanosphaera pluvialis Cohn

Volvox aureus Ehr.

Volvox globator L. emend. Ehr.

\section{TETRASPORALES}

\section{AS IEROCOCCACEAE}

Asterococcus limneticus G.M. Smith

Asterococcus superbus (Cienk.) Scherffel

Chlamydocapsa ampla (Kütz.) Fott

Chlanydocapsa bacillus (Teiling) Fott

Chlamydocapsa planctonica (W. et G.S.West) Fott

Gloeococcus mucosus $A$. Br.
Pseudosphaerocystis lacustris (Lemai.) Nováková

CHLORANGIELLACEAE

Chlorangiella pygmaea (Ehr.) Silva

Chlorangiopsis epizootica (Korš.) Korš.

Stylosphaeridium chlorangielloides Fott NAUTOCOCCACEAE

Cyanoptyche gloeocystiformis Pascher 
TE TRASPORACEAE

Apiocystis braunianä Näg.

Apiocystis brauniana Näg. var. caput-medusae Bohlin (4)

Chaetopeltis orbicularis Berthold

Gloechaete wittrockiana Lagerheim

Palmella miniata Leibl. (5) (6)

Palmella mucosa Kütz.

Palmella protuberans Grev.

Paulschulzia pseudovolvox (Schulz) Skuja
Paulschulzia tenera (Korš.) Lund

Placosphaera cavanillesiana González Guerrero (7)

Schizochlamys gelatinosa A.Br.

Tetraspora gelatinosa (Wahlb.) C.A. Ag.

Tetraspora lemmermannii Fott

Tetraspora limetica W. et G.S.West

Tetraspora lubrica (Roth) C.A.Ag.

Tetraspora lubrica (Roth) C.A.Ag. var. lacunosa Chauv.

\section{CHLOROCOCCALES}

\section{COCCOMIXACEAE}

Coccomyxa confluens (Kütz.) Fott

Cocconya lacustris (Chodat) Pascher

Elakatothrix gelatinosa Wille

Elakatothrix gelatinosa Wille

f. biplex Nyg.

Elakatothrix genevensis (Reverdin) Hindak CHLOROCOCCACEAE

Ankyra ancora (G.M.Smith) Fott

Bracteacoccus minor (Chodat) Petrová

Characium acuminatum $\mathrm{A} . \mathrm{Br}$.

Characium angustum $\mathrm{A} . \mathrm{Br}$.

Characium ensiforme Hermann

Characium orissicum Philippose

Characium ornithocephalum A.Br.

var. longisetum Ettl

Characium ornithocephalum A.Br.

var. pringsheimii (A.Br.) Kom.

Characium sieboldii A.Br.

Characium strictum A.Br.

Characium subsessile (Wolle) de Toni (4)

Chlorococcum botryoides Moewus

Chlorococcum humicola (Näg.) Rabh.

Chlorococcum infusionum (Schrank) Menegh.

Chlorococcum minutum Starr

Chlorochytriu facciolae (Borzi) Bristol

(8)

Chlorochytrium lemnae Cohn

Desmatractum bipyramidatu (Chodat) Pascher Hydrianum ovale Rabh.

Korshikoviella gracilipes (Lambert) Silva

Korshikoviella limetica (Lemm.) Silva

Pseudocharaciun obtusum (A.Br.)Petry-Hesse

Rhopalosen cylindricus (Lambert) Fott

Schroederia setigera (Schroeder) Lemm.

Tetraedron caudatum (Corda) Hansgirg

Tetraedron hastatum (Reinsch) Hansgirg var. palatinum (9)

Tetraedron lunula (Reinsch) Hansgirg (10)

Tetraedron minimum ( $A . B r$.) Hansgirg

Tetraedron minimum (A.Br.) Hansgirg

var. scrobiculatum Lagerheim

Tetraedron pentaedricum W. et G.S. West
Tetraedron quadratum (Reinsch) Hansgirg

f. minus-obtusum Reinsch (11)

Tetraedron regulare Kütz.

Tetraedron triangulare Korš.

Trebouxia anticipata Ahmadjian ex Arch.

DICTYOSPHAERIACEAE

Botryococcus braunii Kütz.

Botryosphaerella sudetica (Lem.) Silva

Dictyosphaerium ehrenbergianu» Näg.

Dictyosphaerium elegans Bachmann

Dictyosphaerium pulchellum Wood

Dictyosphaerium pulchellum Wood

var. minutum Defl.

Dimorphococcus lunatus A.Br.

Lobocystis planctonica (Tiffany et Ahlstrom) Fott

Quadricoccus verrucosus Fott

HYDRODICIYACEAE

Euastropsis richteri (Schmidle) Lagerheim Hydrodictyon reticulatum (L.) Lagerheim

Pediastrum angulosum (Ehr.) Menegh.

Pediastrum biradiatum Meyen

Pediastrum boryanum (Turpin) Menegh.

Pediastrum boryanum (Iurpin) Menegh. var. cornutum (Racib.) Sulek

Pediastrum boryanum (Turpin) Menegh. var. longicorne Reinsch

Pediastrum braunii Wartmann

Pediastrum conchense González Guerrero

(12)

Pediastrum duplex Meyen

Pediastrum integrum Näg.

Pediastrum integrum Näg. var. granulata Racib. (13)

Pediastrum integrum Näg. var. scutum Racib. (14)

Pediastrum kawraiskyi Schmidle

Pediastrum muticum Kütz. var. inerme Racib. (15)

Pediastrum sculptatum G.M. Smith

Pediastrum selenaea Kütz. (16)

Pediastrum simplex Meyen

Pediastrum simplex Meyen var. annulatum Chodat (17) 
Pediastrum tetras (Ehr.) Ralfs 00CYSIACEAE

Ankistrodesmus bibraianus (Reinsch) Korš.

Ankistrodesmus convolutus Corda var. minutum (Näg.) Rabh. (18)

Ankistrodesmus falcatus (Corda) Ralfs

Ankistrodesmus falcatus (Corda) Ralfs var. mirabile W. et G.S. West (19)

Ankistrodesmus fusiformis Corda sensu Korš. Ankistrodesmus gelifactum (Chodat) Bourr. (20)

Ankistrodesuus gracilis (Reinsch) Korš.

Ankistrodesmus longissimus (Lemm.) Wille var. septatum Chodat (21)

Ankistrodeswus nanoselene Skuja (22)

Ankistrodesmus spiralis (Turner) Lemm.

Ankistrodesmus stipitatus (Chodat) Komárková-Legnerová

Closteriopsis longissima (Lemm.) Lemm.

Closteriospira lemanensis Reverdin

Chlorella conductrix (Brandt) Beij. (23)

Chlorella miniata (Näg.) Oltmanns (24)

Chlorella sacharophila (Krüger) Migula var. ellipsoidea (Gerneck) Fott et Nováková

Chlorella vulgaris Beij.

Chlorella zofingensis Dönz.

Choricystis minor (Skuja) Fott

var. minor Fott

Eremosphaera gigas (Arch.) Fott et Kalina

Eremosphaera viridis de Bary

Glaucocystis duplex Prescott (25)

Glaucocystis nostochinearum Itzigsohn Gloeotaenium loitlesbergerianum Hansgirg Hyaloraphidiun contortu Pascher et Korš. Keratococcus bicaudatus (A.Br.) Boye-Petersen

Keratococcus mucicola (Hust.) Hindak

Kirchneriella aperta Teiling

Kirchneriella contorta (Schmidle) Bohlin

Kirchneriella contorta (Schmidle) Bohlin var. gracillima (Bohlin) Chodat

Kirchneriella dianae (Bohlin) Comas

Kirchneriella lunaris (Kirchner) Moebius

Kirchneriella obesa (W.West) Schmidle

Kirchneriella obesa (W.West) Schmidle var. pygmaea W. et G.S. West (26)

Lagerheimia balatonica (Scherfell) Hindak (27)

Lagerheiaia ciliata (Lagerheim) Chodat Lagerheimia citriformis (Snow) Collins Lagerheimia genevensis (Chodat) Chodat Lagerheimia longiseta (Lemm.) Printz Lagerheimia quadriseta (Lemm.) G.M.Smith Lagerheimia subsalsa Lemm.

Lagerheimia wratislaviensis Schroeder Monoraphidium contortum (Thuret) Komárková - Legnerová

Monoraphidium convolutum (Corda) Komárková -Legnerová
Monoraphidium griffithii (Berkel.) Komárková-Legnerová

Monoraphidium irregulare (G.M.Smith) Komárková-Legnerová

Monoraphidium komarkovae Nyg.

Monoraphidium minutum (Näg.) Komárková-

Legnerová

Monoraphidium tortile (W. et G.S. West) Komárková-Legnerová

Nephrocytium agardhianum Näg.

Nephrocytium limneticum (G.M.Smith) G.M. Smith

Nephrocytium lunatum W. West

Nephrocytium minutum (4)

Nephrochlamys subsolitaria (G.S. West) Korš. Docystis borgei Snow

0ocystis elliptica W. West (28)

Docystis lacustris Chodat

0ocystis marssonii Lemm.

0ocystis naegelii $\mathrm{A} . \mathrm{Br}$.

var. incrassata Lemm. (29)

0ocystis nodulosa W. et G.S.West

Docystis novae-semliae Wille

Oocystis parva W. et G.S. West

0ocystis pusilla Hansirg

Docystis solitaria Wittrock in Wittrock et Nordstedt

0ocystis striata González Guerrero (30)

0ocystis submarina Lagerheim

0ocystis submarina Lagerheim

var. variabilis Skuja

Oonephris obesa (W.West) Fott

Quadrigula closterioides (Bohlin) Printz

Quadrigula chodatii (Tann.-Fullem.) G.M. Smith (31)

Quadrigula lacustris (Chodat) G.M.Smith

Quadrigula pfitzeri (Schroeder) Printz

Selenastrum capricornutu Printz

Selenastrum caudatu Lagerheim (4)

Siderocelis coronata Fott

var. coronata Reháková

Sorastrum americanum (Bohlin) Schmidle

Sorastrum bidentatum Reinsch

Sorastrum spinulosum Näg.

Treubaria planctonica (G.M.Smith) Korš.

Treubaria setigera (Arch.) G.M.Smith

Treubaria triappendiculata Bernard

Trochiscia aciculifera (Lagerheim) Hansgirg (32)

Trochiscia aspera (Reinsch) Hansgirg (4)

Irochiscia granulata (Reinsch) Hansgirg

Trochiscia hirta (Reinsch) Hansgirg

Irochiscia pachyderma Hansgirg (4)

Irochiscia reticularis (Reinsch) Hansgirg

Trochiscia zachariasi Lemm.

PALMELLACEAE

Palmodictyon varium (Näg.) Lemm.

Palmodictyon viride Kütz.

$\mathrm{Planctococcus} \mathrm{sphaerocystiformis} \mathrm{Korš.}$

Sphaerocystis schroeteri Chodat 
PHYTELIACEAE (34)

Golenkinic radiata Chodat

Micractinium pusillum Fresenius

Phytelios de-tonii González Guerrero

RADIOCOCCACEAE

Radiococcus nimbatus (de Wildemann)

Schmidle

Gloeocystis minuta Willén (37) (36)

Gloeocystis major (4)

Gloeocystis rupestris (Lyngbye) Rabh. Gloeocystis vesiculosa Näg.

SCENEDESMACEAE

Actinastrum fluviatile (Schroeder) Fott

Actinastrum hantzschii Lagerheim

Coelastrum cambricum Archer

Coelastrum cambricum Archer

var. intermedium (Bohlin) G.S.West

Coelastrum cubicum Näg.

Coelastrum microporum Näg.

Coelastrum morus W. et G.S.West

Coelastrum proboscideum Bohlin

Coelastrum proboscideum Bohlin

var. pseudocubicum Schroeder

Coelastrum reticulatum (Dang.) Senn

Coelastrum scabrum Reinsch (38)

Coelastrum sphaericum Näg.

Crucigenia fenestrata (Schmidle) Schmidle

Crucigenia minima (Fitschen) Brunnthaler (39)

Crucigenia quadrata Morren

Crucigenia tetrapedia (Kirchner) W. et G.

S. West

Crucigeniella apiculata (Lemm.) Kom.

Crucigeniella crucifera (Wolle) Kom.

Crucigeniella pulchra (W.et G.S.West) Kom. Crucigeniella rectangularis (Näg.) Kom. Crucigeniella truncata (G.M.Smith) Kom. Dictyastrum mirabile Beck-Mannagetta

Didymogenes palatina Schmidle

Scenedesmus acuminatus (Lagerheim) Chodat

Scenedesmus acutiformis Schroeder

Scenedesmus antennatus Bréb. in Ralfs (40)

Scenedesmus arcuatus Lemm.

Scenedesmus armatus Chodat

Scenedesmus armatus Chodat

var. bicaudatus (Guglielmetti) Chodat

Scenedesmus armatus Chodat

var. spinosus Fritsch et Rich (41)

Scenedesaus bicaudatus Dedus.

Scenedesmus bicaudatus Dedus.

var. brevicaudatus Hortob. (42)

Scenedesmus bijuga (Iurpin) Lagerheim (43)

Scenedesmus bijugatus (Turpin) Kütz.

var. seriatus Chodat (44)

Scenedesmus brasiliensis Bohlin

Scenedesmus brevispina (G.M.Smith) Chodat (40)

Scenedesmus coelastroides Schmidle

Scenedesmus communis Hegewald

Scendesmus costatus Schmidle
Scenedesmus costulatus Chodat

Scenedesmus denticulatus Lagerheim

Scenedesmus denticulatus Lagerheim var. gracilis Playfair (45)

Scenedesmus denticulatus Lagerheim var. linearis Hansgirg

Scenedesmus disciformis (Chodat) Fott et Kom .

Scenedesmus dispar Bréb.

Scenedesmus ecornis (Ralfs) Chodat

var. polymorphus Chodat (46)

Scenedesmus ellipticus Corda

Scenedesmus falcatus Chodat (47)

Scenedesmus granulatus W. et G.S. West

Scenedeswus gutwinskii Chodat

Scenedesmus incrassatulus Bohlin

Scenedesmus inermis (Fott) Hegewald

Scenedesmus intermedius Chodat

Scenedesmus intermedius Chodat var. bicaudatus Hortob.

Scenedesmus longus Meyen var. brevispina G.M. Smith (48)

Scenedesmus lunatus (W.et G.S. West) Chodat

Scenedesmus maximus W. et G.S. West

Scenedesmus microspina Chodat

Scenedesmus minutus (G.S.Smith) Chodat

Scenedesmus nanus Chodat

Scenedesmus obliquus (Iurpin) Kütz.

Scenedesmus obtusus Meyen

Scenedesmus opoliensis Richter

Scenedesmus opoliensis Richter var. contacta Prescott

Scenedesmus opoliensis Richter var. monoensis Chodat

Scenedesmus ovalternus Chodat

Scenedesmus ovalternus Chodat

var. graevenitzii (Bernard) Chodat

Scenedesmus platydiscus (G.M.Smith) Chodat

Scenedesmus protuberans Fritsch et Rich

Scenedesmus quadricauda (Turpin) Bréb. var. setosus (Kirchner) Hansgirg (4y)

Scenedesmus quadrispina Chodat

Scenedesmus sempervirens Chodat

Scenedesmus serratus (Corda) Bohl in

Scenedesmus smithii (50)

Scenedesmus spicatus W. et G.S.West (40)

Scenedesmus spinosus Chodat

Scenedesmus subspicatus Chodat

Scenedesmus tetradesmiformis (Wolosz.)

Chodat

Tetradesmus wisconsinensis G.M.Smith

Tetrastrum glabrum (Roll) Ahlstorom et

Tiffany

var. hispanica González Guerrero (51)

Tetrastrum staurogeniaeforme (Schroeder) Lemm.

Tetrastrum triangulare (Chodat) Kom.

Westella botryoides (W.West) de Wildemann

Westellopsis linearis Jao

Willea irregularis (Wille) Schmidle 


\section{ULOTRICHALES}

\section{MICROSPORACEAE}

Miscrospora aequabilis Wichmann

Microspora amoena (Kütz.) Rabh.

Microspora awoena (Kütz.) Rabh.

var. gracilis Wichmann

Microspora floccosa (Vaucher) Thuret

Microspora pachyderma (Wille) Lagerheim

Microspora quadrata Hazen

Microspora rufescens (Kütz.) Lagerheim (52)

Microspora stagnoru® (Kütz.) Lagerheim

Microspora tumidula Hazen

ULOIRICHACEAE

Binuclearia tectorum (Kütz.) Beger

Geminella interrupta (Turpin) Lagerheim

Geminella mutabilis (Näg.) Wille

Geminella ordinata (W. et G.S.West) Hee-

ring

Gloeotila mucosa Kütz.

Gloeotila protogenita Kütz.

Gloeotila scopulina (Hazen) Heering

Gloeotilopsis planctonica Iyengar et Phi-

lippose

Heterotrichopsis viridis Iyengar et Kan-

thamma

Klebshormidiu flaccidu A.Br.

Klebshoraidium flaccidu A.Br.

f. aquatica Heering
Klebshormidium rivulare Kütz.

Klebshormidum subtile (Kütz.) Heering

Koliella spirotaenia (G.S.West) Hindak

$\mathrm{Planctonema} \mathrm{lauterborni} \mathrm{Schmidle}$

Radiofilum conjunctivu Schmidle

Radiofilum irregulare (Wille) Brunthaler

Stichococcus bacillaris Näg.

Stichococcus castellanus González Guerrero (53)

Stichococcus chodati (Bial.) Heering

Stichococcus minor Näg.

Stichococcus minutus Grintzesco et Peterfi

Ulothrix aequalis Kütz. (54)

Ulothrix albicans Kütz.

Ulothrix cylindricum Prescott

Ulothrix implexa Kütz.

Ulothrix mucosa Thuret

Ulothrix oscillarina Kütz.

Ulothrix subtilis Kütz.

Ulothrix tenerrima Kuitz.

Ulothrix tenuissima Kütz.

Ulothrix zonata (weber et Mohr) Kutz.

Uronema confervicolum Lagerheim (55)

Uronema elongatum Hodgetts

\section{CHAETOPHORALES}

BORODINELLACEAE

Chlorosarcinopsis angulosa (Corda) Star-

mach

Chlorosarcinopsis minor Herndon

Chlorosphaeropsis alismatis (Klebs) $\mathrm{Vi}$ scher (56)

Chlorosphaeropsis alveolata Herndon

COLEOCHAETACEAE

Coleochaete divergens Pringsheim

Coleochaete irregularis Pringsheim

Coleochaete orbicularis Pringsheim

Coleochaete pulvinata A.Br.

Coleochaete scutata Bréb.

CHAE TOPHORACEAE

Aphanochaete repens A.Br.

Aphanochaete veraiculoides Wolle

Chaetophora elegans (Roth) C.A.Ag.

Chaetophora elegans (Roth) C.A.Ag. var. longipila (Kütz.) Hansgirg (4)

Chaetophora incrassata (Hudson) Hazen

Chaetophora pisiformis (Roth) C.A.Ag.

Chlorocloniu marcei Margalef
Desmococcus vulgaris (Näg.) Brand

Draparnaldia glomerata (Vaucher) C.A.Ag. Draparnaldia glomerata (Vaucher) C.A.Ag. var. biformis Wittrock (4)

Draparnaldia mutabilis (Roth) Cedergen

Ectochaete endophytu (Moebius) Wille

Ectochaete wittrocki (Wille) Lagerheim

Entocladia gracilis Hansgirg

Entocladia viridis Reinke

Epibolium dermaticola Printz

Gloeoplax weberi Schmidle

Gomontia codiolifera (Chodat) Wille (57)

Gongrosira debaryana Rabh.

Gongrosira gaditana González Guerrero

Gongrosira incrustans (Reinsch) Schmidle

Gongrosira pygmaea Kütz.

Gongrosira schidlei Richter

Gongrosira sclerococcus Kütz.

Jaagiella alpicola Vischer

Microthamnion kuetzingianu Näg.

Microthamnion strictissimu Rabh.

Pleurococcus vulgaris (Greville) Menegh. (58) 
Protoderna pax-augustana González Guerrero Protoderma viride Kütz.

Stigeoclonium amoenum Kütz.

Stigeoclonium farctum Berthold (59)

Stigeoclonium lubricum (Dillw.) Kütz. Stigeoclonium nanum (Dillw.) Kütz.

Stigeoclonium subsecundu Kütz.

Stigeoclonium subsecundun Kütz. var. javanicum Richter (60)

Stigeoclonium tenue (C.A.Ag.) Kütz.
Stigeoclonium variabile (Näg.) Nurul Islam Thamniochaete frutex Margalef

CHAETOSPHAERIDIACEAE

Conochaete comosa Klebahn

Chaetosphaeridiu globosum (Nordstedt)

Klebahn

Chaetosphaeridium minus Hansgirg (4)

Chaetosphaeridium pringsheimii Klebahn DICRANOCHAE TACEAE

Dicranochaete reniformis Hieronymus (61)

\section{OEDOGONIALES}

\section{OEDOGONIACEAE}

Bulbochaete brebissonii Kütz. Bulbochaete crassiuscula Nordstedt Bulbochaete debaryana Wittrock et Lundell Bulbochaete grovae Margalef

Bulbochaete intermedia de Bary var. depressa Wittrock

Bulbochaete minor A.Br.

Bulbochaete mirabilis Wittrock Bulbochaete monile Wittrock et Lundell Bulbochaete nordstedtii Wittrock var. minor Woronichin

Bulbochaete pygmaea Pringsheim Bulbochaete pyrulum Lundell

Bulbochaete rectangularis Wittrock Bulbochaete resendeana Lacerda Oedogonium acmandrium Elfving Oedogonium acrosporu De Bary 0edogonium acrosporum De Bary var. floridense Wolle

Oedogonium anomalum Hirn Oedogonium areolatum Lagerheim Oedogonium borisianu (Le Clerc) wittrock Oedogonium boscii (Le Clerc) Wittrock 0edogonium braunii (Kütz.) Pringsheim Oedogonium capillare (L.) Kütz. Oedogonium capillare (L.) Kütz. var. stagnale (Kütz., Wittrock) Hirn Oedogonium capilliforme Kütz., Wittrock Oedogoniu cardiacum (Hassall) Wittrock Oedogoniu cardiacum (Hassall) Wittrock var. carbonicumittrock

Oedogonium cardiacum (Hassall) Wittrock var. explens Hallas

Oedogonium carrissoi Lacerda

Oedogonium ciliatum (Hassall) Pringsheim

Oedogonium circumplicatum Margalef.

Oedogonium concatenatum (Hassall) Wittrock Oedogoniu concatenatu (Hassall) Wittrock $f$. hutchinsiae (Wittrock) Hirn

Oedogoniu crenulatu Wittrock var. gracilis (Nordstedt) Hirn Oedogonium crispum (Hassall) Wittrock
Oedogonium crispum (Hassall) Wittrock var. gracilescens Wittrock Dedogonium cryptoporu Wittrock Oedogoniu cymatosporum Wittrock et Nordstedt

Oedogonium detonii González Guerrero Oedogonium echinospermu A.Br. Oedogonium fonticola A.Br.

Oedogoniu fragile Wittrock

Oedogoniu gallaecicu Margalef Oedogonium giganteum Kütz. Oedogonium gracilius Iiffany Oedogonium gunnii Wittrock Oedogoniu hians Nordstedt et Hirn Oedogonium hirnii Gutwinski Oedogonium howardi G.S. West Oedogonium intermediu Wittrock Oedogonium itzigsohnii (De Bary) Hirn Oedogonium itzigsohnii (De Bary) Hirn var. minus (W. West) Hirn 0edogonium lageniforme Hirn Oedogoniu landsboroughi (Hassall) Wit trock

Oedogonium leiriense Lacerda 0edogonium longatum Kütz. 0edogonium lusitanicum Lacerda Oedogonium macrandriu Wittrock 0edogonium macrandrium Wittrock var. propinquum (Wittrock) Hirn

Oedogonium minus Wittrock

Oedogonium mitratum Hirn

Oedogoniu nodulosum Wittrock

Oedogonium obesum (Wittrock) Hirn

Dedogonium oryzae Wittrock

Oedogonium paucocostatum Tiffany

Oedogonium petri Wittrock

Oedogonium pisanum Wittrock

Oedogonium plusiosporum Wittrock

Oedogonium pluviale Nordstedt

Oedogonium poecilosporu Nordstedt et Hirn Oedogonium porrectum Nordstedt et Hirn var. gracilior Margalef

0edogonium pringsheimi Cramer, Wittrock 


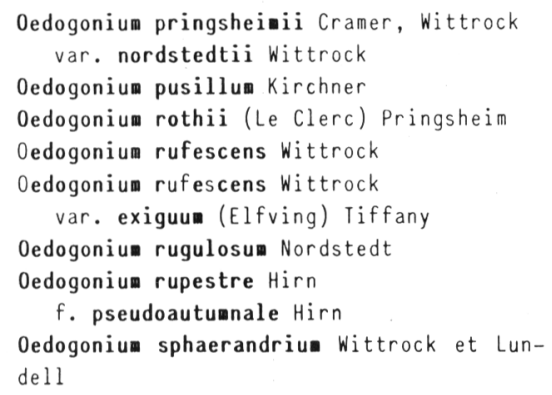

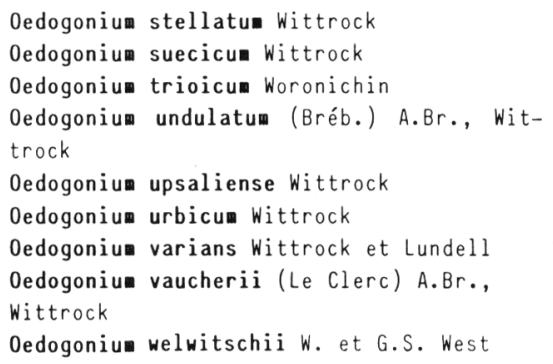

\section{TRENTEPOHLIALES}

\section{TRENTEPOHL IACEAE}

Irentepohlia aurea (L.) Martius
Trentepohlia jolithus (L.) Wallr.

Trentepohlia umbrina (Kütz.) Bornet

\section{SPHAEROPLEALES}

\section{SPHAEROPLEACEAE}

Sphaeroplea annulina (Roth) C.A.Ag.

Sphaeroplea annulina (Roth) C.A.Ag.

var. crassisepta Heinricher
Sphaeroplea leiblenii Kütz.

\section{ULVALES}

MONOSTROMATACEAE

Monostroma bullosum Thuret (62)

SCHIZOMERIDACEAE

Schizomeris leiblenii Kütz.

ULVACEAE

Enteromorpha compressa Greville

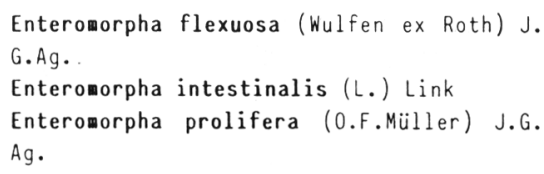

\section{SIPHONOCLADALES}

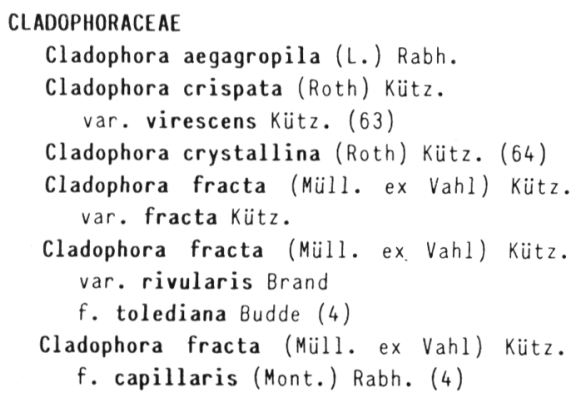

Cladophora globulina Kütz.

Cladophora glomerata (L.) Kütz. var. glowerata (L.) Kütz.

Cladophora glomerata (L.) Kütz. var. crassior (C.A.Ag.) Hoek

Cladophora glomerata (L.) Kütz. $f$. australis (4)

Cladophora rivularis (L.) Hoek

Cladophora sericea (Huds.) Kütz.

Cladophora sericea (Huds.) Kütz. f. aquae-dulcis Areschoug (4) Chaetomorpha aerea (Dillw.) Kütz. 
Chaetomorpha crassa (C.A.Ag.) Kütz. Chaetomorpha herbipolensis Lagerheim Chaetomorpha linum (O.F.Müller) Kütz. Chaetomorpha littorea Harvey Rhizoclonium flavicans (Jürg.) Rabh. Rhizocloniu fontinale Kütz. Rhizoclonium hieroglyphicum (Kütz.) Stockm.
Rhizocloniun hieroglyphicum (Kütz.) Stockm. var. crispum (Kütz.) Rabh.

Rhizoclonium hieroglyphicum (Kütz.) Stockm. var. longiarticulatum Wille

Rhizocloniu lubricu Setchell et Gardner Rhizoclinium ripariu (Roth) Harvey

\section{DUDOSAS PARA ESPAÑA}

\author{
Coelastrum verrucosum (Reinsch) Reinsch \\ (65) \\ Coleochaete soluta (Bréb.) Pringsheim (66) \\ Chlamydomonas intermedia Chodat (66) \\ Chlamydomonas megalis Bis et Bold (67) \\ Chlanydomas steinii Gorosch. (66) \\ Chlorella conglomerata (Artari) Oltmanns \\ (68) \\ Chlorella howosphaera Skuja (69) \\ Chlorococcu botryoides Moewus \\ var. nidulans Hansgirg (70) \\ Chlorolobion braunii (Näg.) Kom. (71) \\ Dictyosphaerium subsolitariun van Goor \\ (72) \\ Dispora crucigenioides Printz (73)
}

\author{
Gongrosira tretepohliopsis Schmidle (74) \\ Lobocystis dichotoma \\ var. mucosa Bourr. (75) \\ Neochloris conjuncta Archibald (67) \\ Oedogonium nanum Wittrock, Tiffany (76) \\ Oedogonium nudulatu (Bréb.) A.Br. (77) \\ Oedogoniu pisanum Wittrock (76) \\ Oedogonium richterianu Lemm. (66) \\ Scenedesmus acutiformis \\ var. tricostatus Chodat (78) \\ Scenedesmus brasiliensis \\ var. norvegicus Printz $(66,79)$ \\ Scenedesmus serratus \\ f. minor chodat (79) \\ Ulothrix pseudoflacca Wille $(4,80)$
}

\section{SINONIMIAS}

Actinastrun hantzschii var. fluviatilis Schroeder = A. fluviatile (Schroeder) Fott Ankistrodesus acicularis (A.Br.) Korł. = Monoraphidium griffithii (Berkel.) KomárkovaLegnerová

A. closterioides (Bohlin) Printz = Quadrigula closterioides (Bohlin) Printz

A. chodatii (Tanner-Fullem.) Brunn. = O. chodatii (Tanner-Fullem.) G.M.Smith

A. falcatus var. acicularis (A.Br.) G.S. West = Monoraphidium griffithii (Berkel.) Komárková-Legnerová

A. falcatus var. duplex (Kütz.) G.S.West = M. contortum (Thuret) Komárkova-Legnerová

A. falcatus var. fasciculatus Corda = A. falcatus (Corda) Ralfs

A. falcatus var. radiatus (Chodat) Lemm. = A. falcatus (Corda) Ralfs

A. falcatus var. setiformis Nyg. f. elongatus Nyg. = Monoraphidium komarkovae Nyg.

A. falcatus var. spiralis G.S. West = A. spiralis (Turn.) Lemm.

A. falcatus var. spirilliformis G.S.West = Monoraphidium contortu (Thuret) KomárkováLegnerová

A. falcatus var. stipitatus Lemm. = A. stipitatus (Chodat) Komárková-Legnerová

A. lacustris (Chodat) Ostenf. = Elakatothrix genevensis (Reverdin) Hindak

A. longissimus (Lemm.) Wille = Closteriopsis longissima (Lemm.) Lemm.

A. mucicola (Hust.) Teiling = Keratococcus mucicola (Hust.) Hindak

A. septatus Chodat et Oettli = Chlorolobion braunii (Näg.) Kom.

A. setigerus (Schroeder) G.S.West = Schroederia setigera (Schroeder) Lemm.

A. spirilliformis G.S. West = Monoraphidiu contortum (Thuret) Komárková-Legnerová

A. spirotaenia G.S. West = Koliella spirotaenia (G.S.West) Hindak

Aphanochaete hyalothecae (Hansgirg) Schmidle = A. vermiculoides Wolle

Binuclearia tatrana Wittrock $=$ B. tectorum (Kütz.) Beger

Botryococcus sudeticus Lemm. = Botryosphaerella sudetica (Lemm.) Silva

Cladophora crispata (Roth) Kütz. = C. glomerata var. crassior (C.A.Ag.) Hoek

C. crispata var. arenaria Kütz. = C. glomerata var. glomerata (L.) Kütz. 
C. crispata var. genuina (Kütz.) Rabh. = C. glowerata var. crassior (C.A.Ag.) Hoek

c. cristata $f$. maura Lewin = C. glomerata var. glomerata

C. elongata Kütz. = C. glomerata var. glomerata

C. fasciculata Kütz. = C. glomerata var. glomerata

C. fracta var. lacustris (Kütz.) Brand = C. globulina Kütz.

C. fracta subsp. leptoderma Lewin = C. glomerata var. crassior (C.A.Ag.) Hoek

C. fracta var. normalis Rabh. = C. fracta var. fracta

C. fracta var. prolifera (C.A.Ag.) = C. fracta var. fracta

C. fracta var. rivularis Brand = C. rivularis (L.) Hoek

C. fracta var. terrestris Kütz. = C. fracta var. fracta

C. glomerata var. arbuscula Rabh. = C. glomerata var. glomerata

C. gossypina (Oraparnand ex Kütz.) Kütz. = C. glomerata var. crassior (C.A.Ag.) Hoek

C. insignis var. fluviatilis Kütz. = C. glonerata var. glomerata

C. insignis var. rivularis Wittrock et Nordstedt = C. rivularis (L.) Hoek

C. lacustris Kütz. = C. globulina Kütz.

C. nitida Kütz. = C. sericea (Huds.) Kütz.

C. nitidissima Menegh. = C. sericea (Huds.) Kütz.

Cocconixa dispar Schmidle = Cocconixa confluens (Kütz.) Fott

C. mon Skuja = Choricystis minor (Skuja) Fott var. minor

Conferva capillaris $L$. = 0edogoniun capillare (L.) Kütz.

C. fontinalis Kütz. = Rhizoclonium hieroglyphicum (Kütz.) Stockm.

C. rivularis L. = Cladophora rivularis (L.) Hoek

Crucigenia apiculata (Lemm.) Schmidle = Crucigeniella apiculata (Lemm.) Kom.

C. irregularis Wille = Willea irregularis (Wille) Schmidle

C. rectangularis (Näg.) Gay = Crucigeniella rectangularis (Näg.) Kom.

C. triangularis Chodat = Tetrastrun triangulare (Chodat) Kom .

C. truncata G.M.Smith = Crucigeniella truncata (G.M.Smith) Kom.

Cyanoptyche gloeocystis Pascher = Cyanoptyche gloeocystiformis Pascher

Chaetomorpha vasta Kütz. = Chaetomorpha aerea (Dillw.) Kütz.

Characiochloris epizootica (Korš.) Pascher = Chlorangiopsis epizootica (Korš.) Korš.

Characiun anbiguun Hermann ex Rabh. = Characiun ensiforme Hermann

Ch. apiculatu Rabh. = Ch. angustun $\mathrm{A} \cdot \mathrm{Br}$.

Ch. braunii Bruegger $=$ Ch. acuninatun $\mathrm{A} \cdot \mathrm{Br}$.

Ch. cylindricum Lambert $=$ Rhopalosen cylindricus (Lambert) Fott

Ch. gracilipes Lambert = Korshikoviella gracilipes (Lambert) Silva

Ch. lieneticun Lemm. = K. limnetica (Lemm.) Silva

Ch. inutu $A \cdot B r$. = Ch. ensiforme Hermann

Ch. obtusu $A . B r$. = Pseudocharaciun obtusun $(A . B r$.) Petry-Hesse

Ch. pringsheimii $A \cdot B r$. = Ch. ornithocephalu var. pringsheimii (A.Br.) Kom.

Ch. rabenhorstii de Toni = Hydrianum ovale Rabh.

Ch. setiferu Cortés = Ch. ornithocephalun var. longisetun Ettl

Chlanydococcus pluvialis Flot. = Haematococcus pluvialis Flot. emend. Wille

Chlanydomas acidophila Nyg. = Chloromas acidophila (Nyg.) Gerloff et Ettl

Ch. pluristigma Bristol = Chlanydomonas snowiae var. pluristigma (Bristol) Gerloff

Chlorangium stentorinum (Ehr.) Stein = Chlorangiella pygmaea (Ehr.) Silva

Chlorella ellipsoidea Gerneck = Chlorella saccharophila var. ellipsoidea (Gerneck) Fott et Nováková

Ch. pyrenoidosa Chick $=\mathrm{Ch}$. vulgaris Beij.

Chlorococcu infusionu var. roemerianum (Kütz.) Hansgirg = Chlorococcum infusionum (Schrank) Menegh.

Chlorosphaera alismatis Klebs = Chlorosphaeropsis alismatis (Klebs) Vischer

Ch. angulosa (Corda) Klebs = Chlorosarcinopsis angulosa (Corda) Starmach

Chodatella Lemm. 1898 = Lagerheimia Chodat 1895

Chodatella citriforais Snow = Lagerheimia longiseta (Lemm.) Printz

Ch. quadriseta Lemm. = L. genevensis (Chodat) Chodat

Dactylococcopsis acicularis Lemm. = Monoraphidium griffithii (Berkel.) Komárková-Legnerová

Dictyosphaerium simplex Skuja = D. subsolitarium van Goor

Draparnaldia nana Hassall = Stigeoclonium nanu (Dillw.) Kütz.

D. plunosa C.A.Ag. = D. utabilis (Roth) Cedergren

D. tenuis C.A.Ag. = Stigeocloniun tenue (C.A.Ag.) Kütz. 
Endoderma gracile (Hansgirg) de Toni = Entocladia gracilis Hansgirg

E. wittrockii (Wille) Lagerheim = Ectochaete wittrocki (Wille) Lagerheim

E. viride (Reinke) Lagerheim = Entocladia viridis Reinke

Enteromorpha salina Kütz. = Enteromorpha flexuosa (Wulfen ex Roth) J.G.Ag.

E. tubulosa Kütz. = E. flexuosa (Wulfen ex Roth) J.G.Ag.

Endorina charkowiensis (Korš.) Pascher = Pandorina charkowiensis Korš.

E. stagnalis Wolle = E. elegans Ehr.

Gemellicystis neglecta Teiling emend. Skuja = Pseudosphaerocystis lacustris (Lemm.) Nováková

Gloeocapsa polydermatica Kütz. = Gloeocystis rupestris (Lyngbye) Rabh. ( 81 )

Gloeococcus schroeteri (Chodat) Lemm. = Spaherocystis schroeteri Chodat

Gloeocystis gigas (Kütz.) Lagerheim = Chlamydocapsa planctonica (W. et G.S.West) Fott

G. planctonica (W. et G.S.West) Lemm. = Ch. planctonica (W. et G.S.

West ) Fott

Gongrosira koppei Budde = Gongrosira schmidlei Richter

G. viridis Kütz. = G. sclerococcus Kütz.

Haematococcus lacustris (Girod.) Rostaf. = H. pluvialis Fott. emend. Wille

Hormidium Kütz. 1843, Clorhormidium Fott 1960 = Klebshormidium Silva, Mattox et Blackwell 1972

Hormidium gaditanum González Guerrero = Klebshormidium flaccidum A.Br.

Hormiscia flaccida var. antliaria (Kütz.) Hansgirg = K. flaccidum A.Br.

H. implexa (Kütz.) Rabh. = Ulothrix implexa Rabh.

H. subtilis (Kütz.) de $\operatorname{Ton} i=U$. tenerrima Kütz.

H.subtilis var. compacta (Roth) Hansgirg = U. tenerrima Kütz.

H. subtilis var. tenerrima (Kütz.) Kirchner = U. tenerrima Kütz.

H. subtilis var. variabilis (Kütz.) Kirchner, de Toni = U. tenerrima Kütz.

H. zonata (Weber et Mohr) Areschoug = U. zonata (Weber et Mohr) Kütz.

H. zonata var. inaequalis (Kütz.) de Toni $=\mathbf{U}$. zonata (Weber et Mohr) Kütz.

Hydrodictyon utriculatu Roth $=H$. reticulatu $(L$.) Lagerheim

Kirchneriella gracillima Bohlin $=K$. contorta var. gracillima (Bohlin) Chodat

$K$. lunaris var. dianae Bohlin $=K$. dianae (Bohlin) Comas

K. obesa var. aperta (Teiling) Brunn. = K. aperta Teiling

K. subsolitaria G.S. West = Nephrochlanys subsolitaria (G.S. West) Korš.

Lagerheimia wratislaviensis $f$. brevispina Caballero = L. wratislaviensis Schroeder

Lobocystis dichotoma Ihompson = Lobocystis plantonica (Iiffany et Ahlstrom) Fott

Lola lubrica Hamel= Rhizocloniun lubricum Setchell et Gardner

Microspora elegans Hansirg = M. amoena var. gracilis Wichmann

M. fontinalis (Berk.) de Toni = Rhizoclonium hieroglyphicum (Kütz.) Stockm.

Monoraphidiu setiforme (Nyg.) Komárková-Legnerová = M. komarkovae Nyg.

Nephrocytiun closterioides Bohlin = Quadrigula closterioides (Bohlin) Printz

N. naegelii Grun. in Rabh. = N. agardhianu» Näg.

N. obesun W. West $=$ 0onephris obesa (W. West) Fott

Oedogoniu calcareun Cleve var. gaditanu Lewin $=0$. braunii Kütz., Pringsheim

0. hispanicu Lewin $=0$. crispum (Hassall) Wittrock

0. pachydermatosporum Nordstedt $=0$. pringsheimii var. nordstedtii Wittrock

0. stagnale Kütz. var. variabilis Lewin $=0$. anomalum Hirn

0 . subspisanum Lewin $=0$. pisanu Wittrock

0ocystis asymetrica W. et G.S. West $(82)=0$ ocystis solitaria Wittrock in Wittrock et Nords.

0. crassa Wittrock $=0$. solitaria Wittrock in Wittrock et Nordstedt

0 . crassa var. marssonii (Lemm.) Printz $=0$. marssonii Lemm.

0. crassa var. mornor Nyg. $=0$. marssonii Lemm.

0. elliptica $f$. minor $W$. West $=0$. elliptica W. West

0. gigas Archer = Eremosphaera gigas (Archer) Fott et Kalina

0. novae-semliae var. tuberculata Schmidle = 0ocystis novae-semliae Wille

0. pseudocoronata Korš. = Siderocelis coronata var. coronata Reháková

0 . rupestris Kirchner $=0$. solitaria Wittrock

0 . solitaria var. notabile W. et G.S. West $=0$. solitaria Wittrock

Ourococcus bicaudatus ( $\mathrm{A} . \mathrm{Br}$.) Grobety = Keratococcus bicaudatus $(\mathrm{A} . \mathrm{Br}$.) Boye-Petersen

Paulschulzia elegans (Woronichin) Fott = P. tenera (Korš.) Lund

Pediastrum angulosum var. araneosum Racib. = Pediastrua angulosum (Ehr.) Menegh. 
P. araneosum (Racib.) G.M.Smith = P. angulosum (Ehr.) Menegh.

P. biradiatu var. longecornutum Gutwinski $=P$. biradiatum Meyen

P. boryanum var. boryanum = P. boryanum (Iurpin) Menegh.

P. boryanua var. brevicorne A.Br. = P. boryanum (Turpin) Menegh.

P. boryanum var. granulatum (Kütz.) A.Br. = P. boryanum (Turpin) Menegh.

P. boryanu var. perforatu (Racib.) Nitardy = P. boryanu (Turpin) Menegh.

P. clathratun (Schroeder) Lemm. = P. simplex Meyen

P. clathratu var. duodenarium (Bailey) Lemm. = P. simplex Meyen

$P$. constrictum Hassall $=P$. boryanum (Iurpin) Menegh.

P. duplex var. clathratum $(A . B r$.$) Lagerheim = P. duplex Meyen$

P. duplex var. cornutun Racib. = P. boryanum var. cornutun (Racib.) Sulek

P. duplex var. gracillimu W. et G.S. West $=$ P. duplex Meyen

P. duplex var. genuinu" (Kirchner) Lagerheim $=$ P. duplex Meyen

$P$. duplex var. reticulatun Lagerheim $=P$. duplex Meyen

P. ehrenbergii (Corda) A.Br. = P. tetras (Ehr.) Ralfs

P. granulatu Kütz. = P. boryanu (Turpin) Menegh.

$P$. longicorne $=P$. boryanum var. longicorne Reinsch

P. muticum Kütz. = P. boryanum (Turpin) Menegh.

P. moticu var. brevicorne Racib. = P. boryanum (Turpin) Menegh.

P. muticu var. longicorne Racib. = P. boryanum var. longicorne Reinsch

P. rotula (Ehr.) Kütz. = P. biradiatum Meyen

P. simplex var. duodenarium (Bailey) Rabh. = P. simplex Meyen

P. simplex var. granulatu Lemm. = P. simplex Meyen

P. simplex var. radians Lemm. = P. simplex Meyen

P. tetras var. excisu (A.Br.) Hansgirg = P. tetras (Ehr.) Ralfs

P. tetras var. tetraodon (Corda) Hansgirg = P. tetras (Ehr.) Ralfs

P. tricornutum Borge $=P$. braunii Wartmann

Protococcus viridis C.A.Ag. = Desmococcus vulgaris (Näg.) Brand

Protodera cavanillesiana González Guerrero = Protoderma viride Kütz.

Radiofilum apiculatu W. et G.S. West = Radiofilum conjunctivu Schmidle

Rhaphidiun convolutun (Corda) Rabh. = Monoraphidiun convolutu (Corda) Komárková-Legnerová

Rhaphidiu fasciculatum Kütz. = Ankistrodesmus fusiformis Corda sensu Korš. (83)

Rhaphidiu polymoephum Fresenius = Monoraphidium griffithii (Berk.) Komárková-Legnerová

Rhizocloniu fontanum Kütz. = Rhizoclonium fontinale Kütz.

R. hieroglyphicu var. riparium (Harvey) Stockm. = R. riparium (Roth) Harvey

R. kochianu= Kütz. = R. flavicans (Jürg.) Rabh.

R. rivulare Kütz. = Cladophora rivularis (L.) Hoek

Richteriella botryoides (Schmidle) Lemm. = Micractiniu pusillum Fresenius

Scenedesmus abundans (Kirchner) Chodat = Scenedesmus sempervirens Chodat

S. abundans var. asymetrica (Schroeder) Morgan = S. subspicatus Chodat

S. acutus Meyen = S. obliquus (Turpin) Kütz. (84)

S. alternans Reinsch $=$ S. obtusus Meyen

S. arcuatus var. platydisca G.M.Smith = S. platydiscus (G.M. Smith) Chodat

S. armatus var. typicus Chodat $=$ S. armatus Chodat

S. bicaudatus (Hansgirg) Chodat $=$ S. bicaudatus Dedus

S. bijuga var. alternans (Reinsch) Borge $=$ S. obtusus Meyen

S. bijugatus (Turpin) Kütz. = S. bijuga (Turpin) Lagerheim

S. bijugatus var. alternans (Reinsch) Hansgirg = S. obtusus Meyen

S. carinatus (Lemm.) Chodat $=$ S. opoliensis Richter

S. costatus var. coelastroides Bohlin = S. costatus Schmidle

S. denticulatus var. biseriatus González Guerrero = S. denticulatus Lagerheim

S. dimorphus (Turpin) Kütz. = S. obliquus (Turpin) Kütz.

S. graevenitzii (Bernard) Margalef = S. ovalternus var. graevenitzii (Bernard) Chodat

S. ecornis (Ralfs) Chodat = S. ellipticus Corda

S. ecornis var. disciformis Chodat $=$ S. disciformis (Chodat) Fott et Kom.

S. incrassatulus Bohlin

S. longispina Chodat $=$ S: armatus Chodat

S. longispina $f$. bicaudatus (Hansgirg) = S. armatus var. bicaudatus (Guglielmetti) Chodat

S. longus Meyen = S. maximus W. et G.S. West

S. longus var. carpetana González Guerrero = S. maximus W. et G.S. West 
S. opoliensis var. abundans $\operatorname{Printz}=\mathrm{S}$. sempervirens Chodat

S. opoliensis var. carinatus Lemm. = S. acuminatus Chodat

S. quadricauda (Turpin) Bréb. = S. communis Hegewald

S. quadricauda $f$. abundans Kirchner = S. sempervirens Chodat

S. quadricauda var. asymetrica Schroeder = S. subspicatus Chodat

S. quadricuada var. dispar (Bréb.) Brunn. = S. dispar Bréb.

S. quadricauda var. longispina (Corda) G.M.Smith = S. armatus Chodat

S. quadricauda var. maximus W. et G.S. West = S. maximus W. et G.S. West

S. quadricauda var. quadrispina (Chodat) G.M.Smith = S. armatus Chodat

S. quadricauda var. westii G.M.Smith = S. armatus Chodat

S. westii (G.M.Smith) Chodat $=$ S. armatus Chodat

S. wisconsinensis (G.M.Smith) Chodat = Ietradesmus wisconsinensis G.M.Smith

Schizochlamys delicatula W. West = Schizochlamydella delicatula (W. West) Korš.

Schroederia setigera var. ancora G.M.Smith = Ankyra ancora (G.M.Smith) Fott

Selenastru bibraianu Reinsch = Ankistrodesmus bibraianus (Reinsch) Korš.

S. gracile Reinsch = A. gracilis (Reinsch) Korš.

S. minutu (Näg.) Collins = Monoraphidium minutum (Näg.) Komárková-Legnerová

S. westii G.M.Smith = Ankistrodesmus gracilis (Reinsch) Korš.

Sphaerella buetschlii Blochmann = Haematococcus buetschlii Blochmann

S. lacustris (Girod.) Wittrock = H. pluvialis Flot. emend. Wille

Sphaerocystis sphaerocystiformis (Korš.) Bourr. = Planctococcus sphaerocystiformis Korš.

Sphaeroplea annulina var. leiblenii (Kütz.) Kirchner = S. leiblenii Kütz.

S. braunii Kütz. emend. Klebahn = S. annulina (Roth) C.A.Ag.

S. crassisepta Klebahn = S. annulina var. crassisepta Heinricher

Stichococcus scopulinus Hazen = Gloeotila scopulina (Hazen) Heering

S. subtilis (Kütz.) Klerck = Klebshormidium subtile (Kütz.) Heering

Stigeoclonium longearticulatum (Hansgirg) Heering = S. subsecundum Kütz.

S. pygmaeum Hansirg = S. variabile (Näg.) Nurul Islam

S. tenue var. irregulare (Kütz.) Rabh. = S. tenue (C.A.Ag.) Kütz.

Tetracoccus botryoides W. West $=$ Westella botryoides (W. West) de Wildemann

Tetraedron caudatu var. incisum Lagerheim = Tetraedron caudatu (Corda) Hansgirg

T. trigonu var. papilliferum (Schroeder) Lemm. = T. triangulare Korš.

Tetrapedia crux-michaeli Reinsch = Crucigenia tetrapedia (Reinsch) W. et G.S. West

Tetraspora explanata C.A.Ag. = Tetraspora gelatinosa (Wahlb.) C.A.Ag.

T. lacunosa Chauv. = I. lubrica var. Lacunosa Chauv.

T. lacustris Lemm. = I. lemmermannii Fott

T. ulvacea Kütz. = T. gelatinosa (Wahlb.) C.A.Ag.

Tetrastrum apiculatum (Lemm.) Schmidle ex Brunn. = Crucigeniella apiculata (Lemm.) Kom.

T. pulloideu Teiling = Crucigeniella pulchra (W. et G.S. West) Kom.

Ulothrix moniliformis Kütz. = U. albicans Kütz.

U. oscillatorina Kütz. = U. oscillarina Kütz.

U. pallide-virens Kütz. = U. tenerrima Kütz.

U. subtilissima Rabh. = U. tenerrima Kütz.

U. variabilis kütz. = U. tenerrima Kütz.

Uronema confervoides = Uronema confervicolum Lagerheim

Westella linearis G.M.Smith = Westellopsis linearis Jao

Zoochlorella conductrix Brandt = Chlorella conductrix (Brandt) Beij.

PRASINOPHYCEAE T. Christensen ex Silva

POLYBLEPHARIDALES

PEDINOMONADACEAE

Scourfieldia complanata G.S. West

Scourfieldia cordifor is Takeda
POL YBLEPHARIDACEAE

Polyblepharides singularis Dang. 


\section{PYRAMINONADALES}

PYRAMINONADACEAE

Pyraminonas inconstans Hodgett
Pyraminonas tetrarhynchus Schmarda

\section{TETRASELMIDALES}

\author{
TE TRASELM IDACEAE \\ Tetraselmis bolosiana (Margalef) Norris, \\ Hori et Chihara (85) \\ Tetraselmis fontiana (Margalef) Norris, \\ Hori et Chihara
}

\author{
Tetraselmis gracilis (Kylin) Butcher
}

Tetraselmis hazenii (Wille) Butcher

DUDOSAS PARA ESPAÑA

Tetraselmis contracta (N.Çarter) Butcher (74)
Tetraselmis subcordiformis (Wille) Butcher (86)

\section{SINONIMIAS}

Platymonas bolosiana Margalef = Tetraselmis bolosiana (Margalef) Norris, Hori et Chihara

P. contracta N.Carter = T. contracta (N.Carter) Butcher

P. Fontiana Margalef = I. fontiana (Margalef) Norris, Hori et Chihara

P. gracilis Kylin = T. gracilis (Kylin) Butcher

P. subcordiformis (Wille) Hazen = I. hazenii (Wille) Butcher

P. willei kylin = T. subcordiformis (Wille) Butcher

\section{NOTAS}

La bibliografía española que aqui se cita puede encontrarse referida en Alvarez Cobelas (1981).

(1) Quizá sea en realidad un taxon de Pteromonas Seligo 1888 (Phacotaceae).

(2) Según Ettl (1975) pertenece al grupo de Ch. debaryana Gorosch., aunque serían precisos nuevos estudios sobre su citología para considerarla como buena especie.

(3) No se cita en la bibliografía consultada por nosotros. ¿Podría ser Ch. planctogloea Skuja o bien Chlamydocapsa planctonica (W. et G.S.West) Fott?. La publicación donde se cita, Margalef et al. (1977), carece de dibujos o descripciones que permitan identificarla.

(4) En la bibliografía del siglo XX no se cita. El estudio al cual pertenece carece de dibujo o descripción que permitan su identificación/sinonimización.

(5) Según Silva (1980) la adscripción de este Género a alguna familia determinada no está clara. La lógica sería a Palmellaceae Decaisne 1842. Komárek \& Fott (1983) lo transfieren al Orden Chlorococcales, aun reconociendo que presenta reproducción por zoosporas. Silva (1980) lo retiene en Tetrasporales. 
(6) van den Hoek (1963a) la considera como un estadio de Chlamydomonas $\mathrm{sp}$.

(7) La situación taxonómica del Género es incierto. Así, Bourrelly (1966) lo sinonimiza a Siderocystopsis Swale 1964 -Chlorococcal-, aunque más tarde (1972) lo reincorpora a las Tetrasporales. Fott (1972) reconoce que su situación taxonómica es poco clara, reteniéndolo en este último Orden. En cuanto a la especie de González Guerrero, Komárek \& Fott (1983) la suponen insuficientemente descrita.

(8) Lewin (1984) propone la transferencia del Género al Orden Chaetophorales Familia Chlorosarcinaceae (=Borodinellaceae, en nuestro esquema), basándose en su tipo de división.

(9) Quizá sea una forma de Treubaria schmidlei (Schroeder) Fott \& Kováčik, de acuerdo con Komárek \& Fott (1983).

(10) En Komárek \& Fott (1983) no se cita, pero podría pertenecer al Género Diplochloris Korš. 1939 (Oocystaceae).

(11) La especie sería próxima a T. minimum, según Kováčik (1975), aunque éste no cita la forma.

(12) Para Sulek (1969) este taxon no está claro. Komárek \& Fott (1983) lo aproximan a P. integrum var. pearsonii (G.S.West) Fritsch.

(13) Ni Sulek (1969) ni Komárek \& Fott (1983) mencionan esta variedad. Bigeard (1934) asimila P. integrum a P. boryanum, pero tampoco cita la variedad.

(14) El taxon no está claro para Sulek (1969). Komárek \& Fott (1983) no lo refieren.

(15) Ibid. (14), aunque el tipo P. muticum Kütz. se sinonimiza en la actualidad a P. boryanum.

(16) Tampoco está claro para Sulek (1969). Bigeard (1934) lo sinonimiza a P. boryanum, mientras que según Komárek \& Fott (1983) sería P. duplex Meyen.

(17) Komárek \& Fott (1983) la indican como sinonimia no confirmada del tipo. Sulek (1969) ignora a qué sinonimizarlo.

(18) Komárková-Legnerová (1969) no la menciona. Probablemente sea Monoraphidiım minutum (Näg.) Komárkova-Legnerová.

(19) No está clara para Komárková-Legnerová (1969), quien vacila en transferirla al Género Closterium (Zygophyceae) o retenerla en las Oocystaceae.

(20) Siguiendo los criterios de Komárková-Legnerová (1969), este taxon debería pasar al grupo de Elakatothrix Wille 1898, aunque ella no lo asigna a ninguna especie concreta ni tampoco lo cita. De acuerdo con Komárek \& Fott (1983), sería próximo al género Pseudoquadrigula Lacoste de Díaz 1973 (Oocystaceae).

(21) Komárková-Legnerová la considera perteneciente al Género Raphidonema Legarheim 1892 (Ulotrichaceae), pero ignora qué especie es. Komárek \& Fott (1983) no la mencionan.

(22) Komárková-Legnerová (1969) considera que es preciso confirmar esta especie mediante cultivos. Komárek \& Fott (1983) la aproximan al Género Chlorolobion Korš. 1953.

(23) Según Komárek \& Fott (1983) se halla insuficientemente descrita, tanto mcrfológica como fisiológicamente.

(24) En la monografía de Fott \& Nováková (1969) no queda claro a qué taxon se sinonimiza éste: ciertas cepas las asimilan a Ch. minutissima Fott et Nováková y otras a Ch. luteoviridis Chodat. Komárek \& Fott (1983) la dan como buena especie. 
(25) Género situado provisionalmente entre las Oocystaceae en tanto que se aclare el carácter del grupo. Consúltese, no obstante, a Schnepf et al. (1966) y a Robinson \& Preston (1971).

(26) La variedad se conoce insuficientemente. Según Komárek \& Fott (1983) debe estar cercana a K. aperta Teiling.

(27) En unó revisión reciente Hindak (1984) asegura que esta especie corresponde en realidad a ciertos estadios de desarrollo de Scenedesmus sp.

(28) Insuficientemente descrita según Reháková (1969), aunque aceptada por Komárek \& Fott (1983).

(29) Reháková (1969) no menciona esta variedad, la cual se hallaría próxima al tipo si seguimos a Komárek \& Fott (1983).

(30) De acuerdo con Komarek \& Fott (1983) las características de este taxon lo alejan del Género Oocystis, pero no indican sinonimia alguna.

(31) Para Komarek \& Fott (1983) es una especie poco clara.

(32) Este Género es una suerte de cajón de sastre que, además, se conoce muy imperfectamente: de muchas de sus especies se ignoran sus distintos estadios ontogénicos, lo cual impide una revisión del mismo.

(33) Tradicionalmente incluido en esta Familia, Palmodictyon ha sido transferido por Komảrek \& Fott (1983) a Radiococcaceae, reconociendo así implicitamente la mala definición de Palmellaceae, Familia que engloba Géneros de Tetrasporales y Chılorococcales en el ING (véanse Silva, 1980, $\mathrm{y}$ nota $\mathrm{n} \div 5$ ).

(34) Silva (1980) incluye aquí a Golenkinia y a Micractinium, Géneros que Komárek \& Fott (1983) sitúan en Golenkiniaceae (Korš.) Kom. 1979 y Micractiniaceae (Brunn.) G.M.Smith 1950, respectivamente. De la primera Familia Silva no habla, mientras que ante la segunda tendría preferencia Phyteliaceae si bien el "status" de Phytelios -el Género que da nombre a la Familia- está muy controvertido (nota siguiente).

(35) Aunque Phytelios podría ser una Golenkinia senescente sin pirenoides, de ser un Género independiente tendría prioridad sobre la alternativa que se le ha propuesto, Golenkiniopsis Korš. 1953, según Komárek \& Fott (1983). En cuanto a la especie de González Guerrero, la ausencia de detalles sobre su contenido celular en la descripción impide su correcta clasificación.

(36) Este Género resulta problemático porque está compuesto por especies de situación taxonómica incierta: unas provenientes de las Cianofíceas y otras de las Tetrasporales. Parece conveniente una revisión pormenorizada del mismo.

(37) Próximo a Coenochloris hindakii Kom. (Radiococcaceae), según Komárek $\&$ Fott (1983):

(38) Diagnosis incompleta, según Sodomková (1972). Komárek \& Fott vacilan en sinonimizarlo a C. stuhlmanii Schmidle, aunque de ser àí C. scabrum tendría prioridad.

(39) Para Komárek (1974) no es una especie clara. Quizá sea, según él, un cenobio joven de Tetrastrum triangulare (Chodat) Kom. En la obra de 1983 no la mencionan.

(40) Hegewald (com. pers.) no sabe a qué asignarlo. Komárek \& Fott (1983) la dan como buena.

(41) Hegewald (com. pers.) vacila en la asignación. Komárek \& Fott (1983) no la citan.

(42) Según Komárek \& Fott (1983) podría ser sinónimo, bien de S. aldavei Hegewald et Schnepf, bien de S. insignis (W. et G.S.West) Chodat. Hegewald (com. pers.), sin embargo, ignora a qué corresponde. 
(43) De acuerdo con Hegewald (com. pers.) este nombre ha sido usado incorrectamente en lugar de S. ellipticus Corda.

(44) Hegewald (com. pers.) no sabe a qué atribuirlo. Para Komárek \& Fott (1983) podría ser una sinonimia de S. linearis Kom:

(45) Según Komárek \& Fott (1983) es S. aculeolatus Reinsch. Hegewald (com. pers.) ignora su posible asignación.

(46) Sinonimia de S. flexuosus (Lemm.) Ahlstrom. Hegewald (com. pers.) asigna S. ecornis a S. ellipticus Corda.

(47) Nombre ilegítimo, según Hegewald (com. pers.) Para Komárek \& Fott (1983) es sinónimo de S. acuminatus var. minor G.M.Smith.

(48) Hegewald (com. pers.) vacila en la sinonimia. Para Komárek \& Fott (1983) sería S. brevispina (G.M.Smith) Chodat.

(49) Hegewald (com. pers.) no sabe a qué sinonimizarlo. Para Komárek \& Fott (1983) podría ser sononimia de S. magnus Meyen.

(50) Puede ser, bien S. smithii Chodat, bien S. smithii Teiling. En el primer caso Hegewald (com. pers.) ignora su adscripción y Komárek \& Fott (1983) no lo mencionan. En el segundo, la especie sería buena para éstos últimos, aunque Hegewald no la refiere.

(51) Por el dibujo que da González Guerrero su variedad no se incluiría en Tetrastrum, ya que este Género presenta las células en contacto y su taxon no. No se cita en las revisiones recientes.

(52) Según Wichmann (1937), insuficientemente descrita.

(53) La especie quizá pudiera ser buena si nos atenemos a las dimensiones celulares, pero la presencia de pirenoide la excluye del Género. Nadie más la menciona.

(54) De acuerdo con Lokhorst \& Vroman (1974a) pertenece al Género Microspora, pero no indican la especie. Tanto para Printz (1964) como para Ramanathan (1964) la especie es buena. Por otro lado, González Fragoso (1893d) menciona Hormiscia kochii Kütz. sin descripción ni dibujo; Hormiscia se ha venido asimilando a Klebshormidium, pero las dimensiones y descripción que da de Toni (1889) para aquel taxon son más propias de Ulothrix, quizá de $U$. aequalis.

(55) Mattox \& Bold (1962) sinonimizan el Género a Ulothrix.

(56) Insuficientemente conocida, según Herndon (1958). Starmach (1972) la incluye aquí, pero con él como autor en lugar de Vischer.

(57) Mal conocida, de acuerdo con Bourrelly (1966). González Guerrero (1960) no aporta descripción ni dibujo.

(58) González Fragoso (1893b, d) menciona Protococcus botryoides Kirchner y P. olivaceus (Rabh.) Hansgirg, respectivamente. Caballero y Villaldea (1929b) cita Chlorococcum olivaceum Rabh. ( $i=$ P. olivaceus?). En la bibliografía actual nadie los cita -no asi de Toni. (1889)-; pudieran pertenecer a Chlorococcum (Chlorococcaceae), aunque siguiendo a Margalef (1952b) y a Brand (1925) es más probable que sean Desmococcus vulgaris (Chaetophoraceae).

(59) Según Nurul Islam (1963), precisa más estudios para su clarificación.

(60) De acuerdo con Nurul Islam (1963) pertenecería al grupo de S. variabile.

(61) De posición taxonómica problemática, otros autores (van de wiel \& Reymond, 1983) aconsejan transferir el Género a las Tetrasporales, aunque recomiendan más estudios previos. 
(62) Otras especies del Género Monostroma que se han citado para las aguas continentales españolas son: M. fuscum (Postels et Ruprecht) Wittrock, M. grevillei (Thuret) Wittrock y M. wittrockii Bornet. Las dos primeras probablemente correspondan a Gayralia oxysperma (Thuret) Vinogradova; además, la presencia de M. (Ulvopsis) grevillei tampoco se ha comprobado para las costas españolas (Gallardo, 1984). En cuanto a M. wittrockii bien pudiera ser G. oxysperma o M. bullosum. Pero para todos estos casos no se ha podido estudiar el material de base, resultando de todo punto insuficientes las descripciones que se dan en la bibliografía española.

(63) van den Hoek (1963b) no sabe a qué asignarla. Quizá sea C. glomerata. Hay otras dos formas citadas en la bibliografía española que él tampoco considera: C. crispata f. natans (en Margalef, 1951) y C. cirspata var. terrestris (en Zubía, 1921). De todos modos, C. crispata ha sido sinonimizada a C. glomerata var. crassior.

(64) van den Hoek (1963b) no sabe si asignarla a C. vagabunda (L.) Hoek o a C. glomerata var. glomerata (L.) Kütz.

(65) En Margalef (1948b). Quizá sea sinonimia de C. sphaericum Näg., siguiendo a Komárek \&’Fott (1983).

(66) En Margalef (1956b).

(67) En Hernández Mariné (1978a).

(68) En Margalef (1947). Según Komárek \& Fott (1983) la especie precisa revisión.

(69) En Noguerol (1979).

(70) En Margalef (1946e).

(71) En Margalef (1944).

(72) En Margalef et al. (1977).

(73) En Margalef (1947).

(74) En Margalef (1953b).

(75) En Rodríguez González (1972). Komárek \& Fott (1983) insinúan que la especie podría pertenecer al Género Quadricoccus Fott 1948.

(76) En Margalef (1950).

(77) En Cela (1915).

(78) En Margalef (1950b).

(79) En Margalef (1948b).

(80) En Margalef (1952b).

(81) Hay otras especies del Género de Cianofíceas Gloeocapsa que podrían pertenecer a las Chlorococcales, según Komárek \& Fott (1983). G. montana Kütz. pudiera sinonimizarse a Coccomixa ○ a Gloeocystis; G. montana var. flavo-aurantia Kütz. (en Margalef, 1951) y G. muralis Kütz. (en Caballero y Villaldea, 1929b) también se hallan excluidas de las Cianofíceas, pero aún no han sido sinonimizadas.

(82) Próxima al Género Ecdysichlamys G.S.West 1912 (Oocystaceae).

(83) En realidad, Komárková-Legnerová (1969) sólo sinonimiza R. fasciculatum Kütz. sensu Näg.

(84) Para Komarek \& Fott (1983) S. acutus Meyen es buena especie.

(85) La especie se halla insuficientemente descrita según Ettl (1983).

(86) En Margalef (1946a, 1949c). 


\section{DEDICATORIA Y AGRADECIMIENTOS}

Dedicamos este trabajo a Mariona Hernández Mariné y agradecemos al Dr. Ebernara Hegewald, sus indicaciones de nomenclatura en el maremagnum de Scenedesmus.

\section{BIBLIOGRAFIA}

ALVAREZ COBELAS, M. -1981- Bibliografía sobre la vegetación de algas españolas de aguas dulces y salobres. Excerpta Bot. Sect. B., 16: 233247.

ALVAREZ COBELAS, M. -1984a- Catálogo de las algas continentales españolas, II. Craspedophyceae, Cryptophyceae, Chrysophyceae, Dinophyceae, Euglenophyceae, Haptophyceae, Phaeophyceae, Rhodophyceae, Xanthophyceae. Acta Bot. Malacitana, 9: 27-40.

ALVAREZ COBELAS, M. -1984b- Catálogo de las algas continentales españolas, III. Zygophyceae Widder 1960. Collect. Bot., 15: 17-37.

ALVAREZ COBELAS, M. \& ESTEVEZ, A. -1982- Catálogo de las algas continentales españolas, I. Diatomophyceae Rabenhorst 1864. Lazaroa, 4: 269285.

ARCHIBALD, P. \& BOLD, H.C. -1970- The genus Chlorococcum Meneghini. Univ. Texas Publ. Bot. 7015 Phycol. Stud., 11: 1-147.

BIGEARD, E. -1934- Les Pediastrum d'Europe. Etude biologique et systématique. Rev. Algol., 7: 1-94, 327-418.

BLIDING, C. -1963- A critical survey of European taxa in Ulvales. Part I: Capsosiphon, Percursaria, Blidingia, Enteromorpha. Opera Bot., 8: $1-160$.

BLIDING, C. -1968- A critical survey of European taxa in Ulvales. Part II: Ulva, Ulvaria, Monostroma, Kornmannia. Bot. Not., 121: 535-629.

BOURRELly, P. -1966- Les algues d'eau douce. I. Algues vertes. Boubée ed. Paris.

BOURRELLY, P. -1972- Les algues d'eau douce. I. Algues vertes. zème edition. Boubée ed. Paris.

BRAND, F. -1925- Analyse der aerophilen Grünalgenanflüge insbesondere der proto-pleurococcoiden Formen. Arch. Protistenk., 52: 265-355.

COX, E.R. \& BOLD, H.C. -1960- Taxonomic investigations of the genus Stigeoclonium. Univ. Texas Publ. Bot. 6618 Phycol. Stud., 7: 7-167.

ETTL, H. -1970- Die Gattung Chloromonas Gobi emend. Wille. Beih. Nova Hedwigia, 34. J. Cramer Verlag. Lehre.

ETTL, H. -1975- Die Gattung Chlamydomonas Ehr. Beih. Nova Hedwigia, 49. J. Cramer Verlag. Vaduz.

ETTL, H. -1979- Die Gattungen Carteria Diesing emend. Francé und Provasoliella A.R. Loeblich. Beih. Nova Hedwigia, 60. J. Cramer Verlag. Vaduz.

ETTL, H. -1983- Chlorophyta I. Phytomonadina. Süsswasserflora von Mitteleuropa. G. Fischer Verlag. Stuttgart.

FOTT, B. -1972- Tetrasporales. Das Phytoplankton des Süsswassers. Schweizerbart'sche Verlagsbuchhandlung. Stuttgart.

FOTT, B. \& NOVAKOVA, M. -1969- A monograph of the genus Chlorella. The freshwater species. Studies in Phycology (B.Fott, ed.): 10-74. Schweizerbart'sche Verlagsbuchhandlung. Stuttgart.

FRITSCH, F.E. -1929- The genus Sphaeroplea. Ann. Bot., 43: 1-26.

GALLARDO, T. -1984- Taxonomía, ecología y fitosociología de las Ulváceas del Noroeste de España. Tesis Doctoral. Fac. Biología. Univ. Complutense. Madrid.

GEMEINHARDT, K. -1939- Oedogoniales. Dr. Rabenhorst's Kryptogamenflora, 12. Akademische Verlagsgessellschaft. Leipzig. 
GONZALVES, E.A. -1981- Oedogoniales. I.C.A.R. New Delhi.

HAMEL, G. -1931- Chlorophycées des côtes françaises. Rev. Algol., 5: 973.

HEGEWALD, E. -1977- Scenedesmus communis Hegewald, a new species and its relation to Scenedesmus quadricauda (Turpin) Bréb. Arch. Hydrobiol. Suppl., 51: 142-155.

HEGEWALD, E. \& SCHNEPF, E. -1979- Geschichte und Stand der Systematik der Grünalgengattung Scenedesmus. Schweiz. Z. Hydrol., 40: 320-342.

HERNDON, W. -1958- Studies on chlorosphaeracean algae from soil. Amer. J. Bot., 45: 298-308.

HINDAK, F. -1963- Systematik der Gattungen Koliella gen. nov. und Raphidonema Lagerh. Nova hedwigia, 6: 95-125.

'HINDAK, F. -1984- Review of the genus Lagerheimia Chod. incl. Chodatella Lemm. (Chlorococcales, Chlorophyceae). Schweiz. Z. Hydrol., 45: 373387.

HOEK, C. VAN DEN -1963a- Nomenclatural tipification of some unicellular and colonial algae. Nova Hedwigia, 6: 277-296.

HOEK, C. VAN DEN -1963b- Revision of the European species of cladophora. E.J. Brill. Leiden.

HUBER-PESTALOZZI, G. -1961- Volvocales. Das Phytoplankton des Süsswassers. Schweizerbart'sche Verlagsbuchhandlung. Stuttgart.

IRVINE, D.E.G. \& JOHN, D. -1985- The systematics of the Green Algae. Academic Press. New York.

IYENGAR, M.O.P. \& DESIKACHARY, T.J. -1981- Volvocales. I.C.A.R. New Delhi.

JAAG, O. -1933- Die Gattung Coccomixa Schmidle. Beitr. Kryptogamenfl. Schweiz, 8: 1-74.

KOMAREK, J. -1974- The morphology and taxonomy of crucigenioid algae (Scenedesmaceae, Chlorococcales). Arch. Protistenk., 116: 1-75.

KOMAREK, J. \& FOTT, B. -1983- Chlorococcales. Das Phytoplankton des Süsswassers. Schweizerbart'sche Verlagsbuchhandlung. Stuttgart.

KOMARKOVA-LEGNEROVA, J. -1969- The systematics and ontogenesis of the genera Ankistrodesmus Corda and Monoraphidium gen. nov. Studies in Phycology (B. Fott, ed.): 75-144. Schweizerbart'sche Verlagsbuchhandlung. Stuttgart.

KOVACIK, L. -1975- Taxonomic review of the genus Tetraedron (Chlorococcales). Arch. Hydrobiol./Suppl., 46:354-391.

LEE, K.W. \& BOLD, H.C. -1974- Characium and some Characium-like algae. Univ. Texas Publ. Bot. 7403 Phycol. Stud., 12: 1-127.

LEMMERMANN, E. \& BRUNNTHALER, J. -1915- Tetrasporales. Protococcales. Die Süsswasserflora Mitteleuropas . G. Fischer Verlag. Jena.

LEWIN, R. A. -1984- Culture and taxonomic status of Chlorochytrium lemnae, a green algae endophyte. Brit. Phycol. J., 19: 107-116.

LOKHORST, G.M. \& VROMAN, M. -1972- Taxonomic study on three freshwater Ulothrix species. Acta Bot. Neerl., 21: 449-480.

LOKHORST, G.M. \& VROMAN, M. -1974a- Taxonomic studies on the genus Ulothrix (Ulotrichales, Chlorophyceae), II. Acta Bot. Neerl., 23: 369398.

LOKHORST, G.M. \& VROMAN, M. -1974b- Taxonomic studies on the genus Ulothrix (Ulotrichales, Chlorophyceae), III. Acta Bot. Neerl., 23: 561602 .

MATTOX, K.R \& BOLD, H.C. -1962- The taxonomy of certain Ulotrichacean algae. Univ. Texas Publ. Bot. 6222 Phycol. Stud., 3: 1-67.

MROZINSKA-WEBB, T. -1969- Oedogoniales. Flora Slodkowodna Polski, 11. Akademia Nauk. Krakow.

NORRIS, R.E. -1980- Prasinophytes. Phytoflagellates (E.R. Cox, ed.). 85145. Elsevier North Holland. Amsterdam.

NORRIS, R.E., HORI, T. \& CHIHARA, M. -1980- Revision of the genus Tetraselmis (class Prasinophyceae). Bot. Mag. Tokyo, 93: 317-339.

NURUL ISLAM, A.K.M. -1963- A revision of the genus Stigeoclonium. Beih. Nova Hedwigia, 10. J. Cramer Verlag. Weinheim. 
PANKOW, H. -1961- Die Algengattung Uronema Lagerst. Arch. Protistenk., 105: $117-130$.

PARKE, M. \& DIXON, P.S. -1976- Check-listof British marine algae. Third revisión. J. Mar. Biol. Assoc. U.K., 56: 527-594.

PHILIPPOSE, M.T. -1967-Chlorococcales. I.C.A.R. New ielhi.

POCOCK, M.A. -1960- Haematococcus in Southern Africa. Trans Roy. Soc. South. Africa, 36: 5-56.

PRINGSHEIM, E.G. -1969- Die Gattungen Chlorogonium und Hyalogonium (Volvocales). Nova Hedwigia, 18: 831-867.

PRINTZ, H. -1964- Die Chaetophoralen der Binnengewässer. Hydrobiologia, 24: $1-377$.

RAMANATHA, K.R. -1964- Ulotrichales. I.C.A.R. New Delhi.

REHAKOVA, H. -1969- Die Variabilität der Arten der Gattung Oocystis A. Braun. Studies in Phycology (B. Fott, ed.): 145-196. Schweizerbart' sche Verlagsbuchhandlung. Stuttgart.

ROBINSON, D.G. \& PRESTON, R.D. -1971- Studies on the fine structure of Glaucocystis nostochinearum Itzigs. II. Membrane morphology and taxonomy. Brit. Phycol. J., 6: 113-128.

ROUND, F.E. -1963- The taxonomy of Chlorophyta, I. Brit. Phycol. Bull., 2: $224-235$.

ROUND, F.E. -1971- The taxonomy of Chlorophyta, II. Brit. Phycol. Bull., 6: 235-264.

SCHNEPF, E., KOCH, W. \& DEICHGRAEBER,G. -1966- Zur Cytologie und taxonomischen Einordnung von Glaucocystis. Arch. Mikrobiol., 55: 149-174.

SILVA, P.C. -1980- Names of Classes and Families of living algae. Regnum Veg., 103.

SMITH, R.L. \& BOLD, H.C. -1966- Investigations on the algal genera Eremosphaera and Oocystis. Univ. Texas Publ. Bot. 6612 Phycol. Stud., 6: 1-121.

SODOMKOVA, M. -1972- Taxonomische Ubersicht der Gattung Coelastrum Näg. Acta Univ. Carol. Biol., 481-512.

STARMACH, K. -1972- Chlorophyta III. Flora Slodkowodna Polski, 10. Akademia Nauk. Warszawa.

SULEK, J. -1969- Taxonomische Übersicht der Gattung Pediastrum Meyen. Studies in Phycology (B. Fott, ed.): 196-261. Schwe zerbart'sche Verlagsbuchhandlung. Stuttgart.

THOMPSON, R.H. -1956- Schizochlamys gelatinosa and Placosphaera opaca. Amer. J. Bot., 43: 665-672.

TONI, J.B. DE -1889- Sylloge Algarum (Chlorophycearum). Vol. I. Patavii.

UHERKOVICH, G. -1966- Die Scenedesmus-Arten Ungarns. Akademiai Kiado. Budapest.

VINOGRADOVA, K.L. -1974- Ul'vovye vodorosli (Chlorophyta) Morej. Izdatel' stovo "Nauka". Leningrado.

WICHMANN, L. -1937- Studien über die durch H-Stück Bau der Membran ausgezeichneten Gattungen Microspora, Binuclearia, Ulot:ichopsis und Tribonema. Pflanzenforschung, 20: 1-110.

WIEL, C.C.M. VAN DE \& REYMOND, O.L. -1983- Ultrastructure and taxonomic position of the green alga Dicranochaete reniformis Hieronymus. Phycologia, 22: 317-324.

Direcciones de los autores: M. ALVAREZ COBELAS, Centro de Investigaciones del Agua (CSIC). La Poveda, Arganda del Rey. Madrid. T. GALlardo GarCiA, Real Jardín Botánico (CSIC), Plaza de Murillo, 2. 28014 Madrid. 